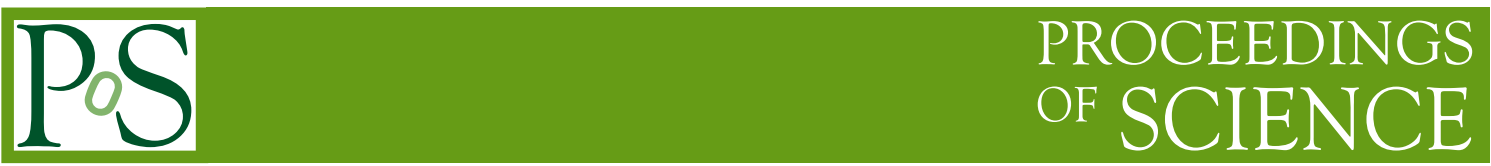

\title{
On the light-front wave functions of quarkonia
}

\author{
Pieter Maris*, Shaoyang Jia, Meijian Li, Yang Li, Shuo Tang and James P. Vary \\ Dept. of Physics and Astronomy, Iowa State University, Ames, IA 50011, USA \\ pmaris@iastate.edu, sjialiastate.edu, meijianleiastate.edu, \\ leeyoung@iastate.edu, tang@iastate.edu, jvary@iastate.edu
}

\begin{abstract}
The light-front wave functions of hadrons allow us to calculate a wide range of physical observables; however, the wave functions themselves cannot be measured. We discuss recent results for quarkonia obtained in basis light-front quantization using an effective Hamiltonian with a confining model in both the transverse and longitudinal directions and with explicit one-gluon exchange. In particular, we focus on the numerical convergence of the basis expansion, as well as the asymptotic behavior of the light-front wave functions. We also illustrate that, for mesons with unequal quark masses, the maxima of the light-front wave functions depend in a non-trivial way on the valence quark-mass difference.
\end{abstract}

Light Cone 2019 - QCD on the light cone: from hadrons to heavy ions - LC2019

16-20 September 2019

Ecole Polytechnique, Palaiseau, France

\footnotetext{
*Speaker.
} 


\section{Confining model for quarkonia}

We use a model for the confining potential between a quark and an anti-quark in a colorsinglet in both the longitudinal and the transverse direction, as described in Refs. [1, 2]. The short-range, high-momentum physics is dominated by one-gluon exchange, which we add to the confining potential. Thus the effective light-front Hamiltonian for a quark and an anti-quark in a flavor-singlet configuration becomes

$$
\begin{aligned}
H_{\mathrm{eff}}= & \frac{\vec{k}_{\perp}^{2}+m_{q}^{2}}{x}+\frac{\vec{k}_{\perp}^{2}+m_{\bar{q}}^{2}}{1-x}+\kappa^{4} \vec{\zeta}_{\perp}^{2}-\frac{\kappa^{4}}{\left(m_{q}+m_{\bar{q}}\right)^{2}} \partial_{x}\left(x(1-x) \partial_{x}\right) \\
& -C_{F} \frac{4 \pi \alpha_{s}\left(Q^{2}\right)}{Q^{2}} \bar{u}_{s^{\prime}}\left(k^{\prime}\right) \gamma_{\mu} u_{s}(k) \bar{v}_{\bar{s}}(\bar{k}) \gamma^{u} v_{\bar{s}^{\prime}}\left(\bar{k}^{\prime}\right),
\end{aligned}
$$

where $m_{q}$ and $m_{\bar{q}}$ are the masses of the quark and anti-quark, $\vec{\zeta}_{\perp} \equiv \sqrt{x(1-x)} \vec{r}_{\perp}$ is Brodsky and de Téramond's holographic variable [3], $\kappa$ is the strength of the confinement, and the longitudinal confinement is described by $\partial_{x} f\left(x, \vec{\zeta}_{\perp}\right)=\partial f\left(x, \vec{\zeta}_{\perp}\right) /\left.\partial x\right|_{\vec{\zeta}}$. The second line corresponds to the onegluon exchange, with $C_{F}=\left(N_{c}^{2}-1\right) /\left(2 N_{c}\right)=4 / 3$ the color factor for the color singlet state, and $Q^{2}=-q^{2}>0$ is the 4-momentum squared carried by the exchanged gluon [2].

The mass spectrum and corresponding light-front wave functions (LFWF) are obtained by diagonalizing the effective light-front Hamiltonian operator (1.1)

$$
H_{\mathrm{eff}}\left|\psi_{h}\left(P, j, m_{j}\right)\right\rangle=M_{h}^{2}\left|\psi_{h}\left(P, j, m_{j}\right)\right\rangle,
$$

where $P=\left(P^{-}, P^{+}, \vec{P}_{\perp}\right)$ is the 4-momentum of the meson, and $j$ and $m_{j}$ are the particle's total angular momentum and magnetic projection, respectively. In the leading $q \bar{q}$ Fock space we have

$$
\begin{aligned}
\left|\psi_{h}\left(P, m_{j}\right)\right\rangle \approx & \sum_{s, \bar{s}} \int_{0}^{1} \frac{\mathrm{d} x}{2 x(1-x)} \int \frac{\mathrm{d}^{2} k_{\perp}}{(2 \pi)^{3}} \psi_{s \bar{s} / h}^{\left(m_{j}\right)}\left(\vec{k}_{\perp}, x\right) \\
& \times \frac{1}{\sqrt{N_{c}}} \sum_{i=1}^{N_{c}} b_{s i}^{\dagger}\left(x P^{+}, \vec{k}_{\perp}+x \vec{P}_{\perp}\right) d_{\overrightarrow{s i}}^{\dagger}\left((1-x) P^{+},-\vec{k}_{\perp}+(1-x) \vec{P}_{\perp}\right)|0\rangle,
\end{aligned}
$$

with $b^{\dagger}$ and $d^{\dagger}$ the quark and anti-quark creation operators and $\psi_{s \bar{s} / h}^{\left(m_{j}\right)}\left(\vec{k}_{\perp}, x\right)$ the valence space LFWF with $s$ and $\bar{s}$ the spin of the quark and antiquark, properly normalized to

$$
\sum_{s, \bar{s}} \int_{0}^{1} \frac{\mathrm{d} x}{2 x(1-x)} \int \frac{\mathrm{d}^{2} k_{\perp}}{(2 \pi)^{3}} \psi_{s \bar{s} / h^{\prime}}^{\left(m_{j}^{\prime}\right) *}\left(\vec{k}_{\perp}, x\right) \psi_{s \bar{s} / h}^{\left(m_{j}\right)}\left(\vec{k}_{\perp}, x\right)=\delta_{h h^{\prime}} \delta_{m_{j}, m_{j}^{\prime}} .
$$

Without the one-gluon exchange, this model can be solved analytically, and the LFWFs can be expressed as a product of a 2-dimensional harmonic oscillator (HO) function $\phi_{n m}$ with strength parameter $\kappa$ and a Jacobi polynomial times power-law factors $\chi_{l}$ in the longitudinal direction [1]. These analytic solutions form a convenient and complete basis for expanding the LFWFs

$$
\psi_{s s^{\prime} / h}\left(\vec{k}_{\perp}, x\right)=\sum_{n, m, l} c_{s s^{\prime} / h}^{n m l} \phi_{n m}\left(\vec{k}_{\perp} / \sqrt{x(1-x)}\right) \chi_{l}(x) .
$$

By expressing our effective Hamiltonian Eq. (1.1) in this basis, Eq. (1.2) becomes a matrix equation for the coefficients $c_{s s^{\prime} / h}^{n m l}$, which we diagonalize numerically. In the limit of a complete (but infinitedimensional) basis, this gives us the exact LFWFs for this Hamiltonian in the leading Fock space. 
This model has been used to compute a number of observables as such as radiative decays and form factors $[4,5]$, well as in diffractive vector meson production [6], showing reasonable agreement with the available experimental data.

\section{Numerical convergence}

The precision of our numerical calculations depends both on the number of basis states that we keep in our expansion, and on the precision of the evaluation of the Hamiltonian matrix elements in this basis. The number of basis states is controlled by the truncation parameters $N_{\max }$ and $L_{\max }$ for the transverse and longitudinal directions, respectively; for simplicity, we keep $N_{\max }=L_{\max }$. The matrix elements are evaluated numerically, using $n_{x}$ and $n_{k}$ integration points for the $x$ and $k_{\perp}$ integrations, respectively; at a minimum we keep $n_{x} \geq L_{\max }$ and $n_{k} \geq N_{\max }$.

\subsection{Meson mass and electroweak decay constants}

The dependence of the masses and decay constants on the basis truncation parameter $N_{\max }$ is shown Fig. 1 for the lowest pseudoscalar and vector $Q \bar{q}$ states. The meson masses appear to converge reasonably well, both with $N_{\max }$ and with the number of integration points $n_{x}$ and $n_{k}$. They do not necessarily converge to the physical values - the parameters we use here were fitted
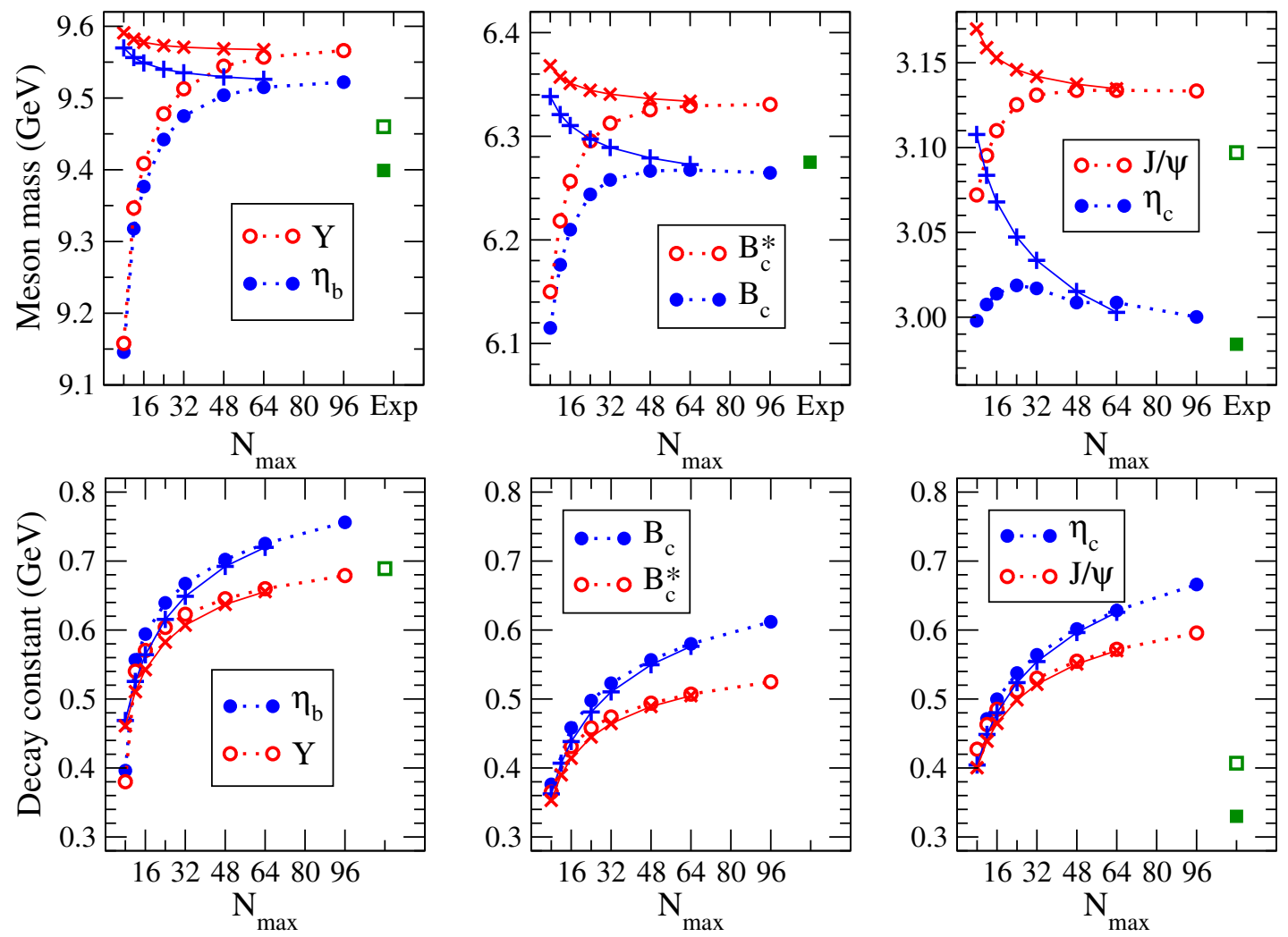

Figure 1: The meson mass (top) and decay constant (bottom) as function of the basis truncation $N_{\max }$ with $n_{x}=n_{k}=2 N_{\max }$ (open and closed circles) and with $n_{x}=192$ and $n_{k}=96$ (plusses and crosses), and experimental values from Ref. [7] (open and closed green squares). 
in Ref. [2] at $N_{\max }=L_{\max }=32$ and $n_{x}=n_{k}=64$ to the lowest 14 and 8 states of bottomonium and charmonium respectively. Note that as we increase $N_{\max }$ while keeping $n_{x}$ and $n_{k}$ fixed, the obtained masses decrease monotonically, in agreement with the variational principle.

On the other hand, the obtained decay constants seem to be (almost) independent of $n_{x}$ and $n_{k}$, but they depend strongly on the basis truncation parameter $N_{\max }$. This suggest that the decay constants (in contrast to the masses) are sensitive to the high-momentum behavior of the LFWF. Indeed, it is known from e.g. the covariant Dyson-Schwinger approach that the integral for the pseudoscalar decay constant over the Bethe-Salpeter amplitude $\chi_{\mathrm{PS}}(k, P)$

$$
f_{\mathrm{PS}}=\frac{Z_{2}}{m_{\mathrm{PS}}^{2}} \int \frac{\mathrm{d}^{4} k}{(2 \pi)^{4}} \operatorname{Tr}\left[\chi_{\mathrm{PS}}(k, P) \gamma_{5} \not P\right]
$$

is potentially divergent. This divergence is absorbed by the wave function renormalization constant $Z_{2}$, rendering a finite result for the physical decay constants [8]. In Ref. [2] we have therefore truncated the corresponding integral in the transverse direction of the light-front formalism

$$
f_{\mathrm{PS}}=\sqrt{2 N_{c}} \int_{0}^{1} \frac{\mathrm{d} x}{\sqrt{x(1-x)}} \int^{\mu} \frac{\mathrm{d}^{2} k_{\perp}}{(2 \pi)^{3}} \psi_{\uparrow \downarrow-\downarrow \uparrow}\left(\vec{k}_{\perp}, x\right)
$$

at an appropriate UV mass scale $\mu \approx 1.7 m_{q}$. In order to address the question of the convergence of the decay constant, we now turn our attention to the asymptotic behavior of the LFWFs.

\subsection{Asymptotics of Light-Front wave functions}

Understanding the ultraviolet (UV) asymptotics of the LFWF as $k_{\perp} \rightarrow \infty$ is crucial for the proper evaluation (with consistent regularization and renormalization as necessary) of observables such as decay constants and elastic and transition form factors. The infrared behavior of the LFWF is dominated by our model for the confining interaction as well as the behavior of the running coupling at small momenta $Q^{2}$. On the other hand, we expect the UV behavior to be dominated by perturbative QCD, and in particular the large- $Q^{2}$ behavior of the one-gluon exchange.

Within our finite basis calculations, the asymptotics can only be well-represented up to the effective UV truncation scale, $\Lambda=\kappa \sqrt{x(1-x) N_{\max }}$, in our transverse basis functions. Indeed,
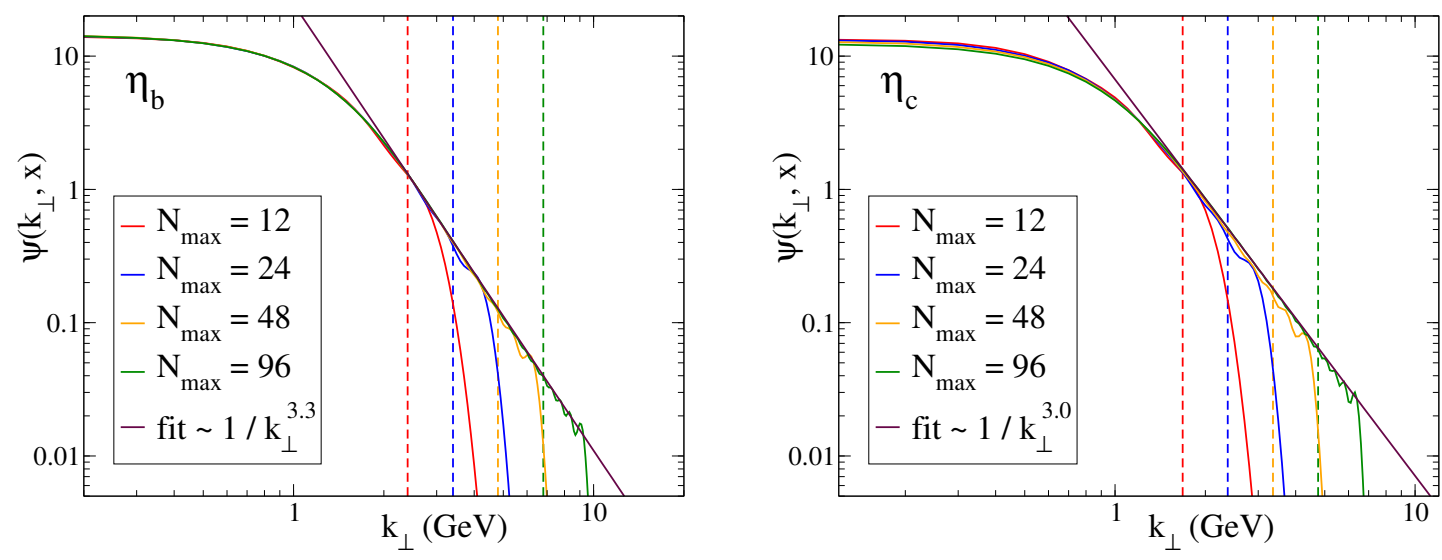

Figure 2: Dominant component $\psi_{\uparrow \downarrow-\downarrow \uparrow}\left(k_{\perp}, x\right)$ of the LFWF for $\eta_{b}$ (left) and $\eta_{c}$ (right) as function of $k_{\perp}$ at $x=\frac{1}{2}$ on a log-log scale. The vertical dashed lines indicate the UV truncation scale $\Lambda$ defined in the text. 
Fig. 2 shows that below this scale $\Lambda$, the LFWFs of the $\eta_{b}$ and $\eta_{c}$ are (almost) independent of the truncation parameter $N_{\max }$, but for $k_{\perp}>\Lambda$ the LFWF starts to oscillate and falls off like a gaussian, as one would expect. For the vector mesons we obtain the same asymptotic behavior. Our calculations suggest that the LFWFs fall off like $1 / k_{\perp}^{3}$ or even faster (possibly with a logarithmic correction), but an expansion in $\mathrm{HO}$ basis functions in the transverse direction is far from the ideal computational method for an accurate determination of the asymptotic behavior of the LFWFs.

\subsection{Asymptotics of the transverse Distribution Amplitude}

Analogous to the (longitudinal) Distribution Amplitudes, which are obtained from the LFWF by integrating over the transverse momenta, one can define Transerse Distribution Amplitudes (TDA) by integrating over $x$

$$
\phi_{\perp}\left(\vec{k}_{\perp}\right)=\sqrt{2 N_{c}} \int_{0}^{1} \frac{\mathrm{d} x}{\sqrt{x(1-x)}} \psi_{\uparrow \downarrow-\downarrow \uparrow}\left(\vec{k}_{\perp}, x\right),
$$

normalized here such that the integral over $\vec{k}_{\perp}$ gives the decay constant

$$
f_{\mathrm{PS}}=\int \frac{\mathrm{d}^{2} \vec{k}_{\perp}}{(2 \pi)^{3}} \phi_{\perp}\left(\vec{k}_{\perp}\right) .
$$

The left panel of Fig. 3 shows $\phi_{\perp}\left(k_{\perp}\right)$ of $\eta_{b}$ for several different basis truncation parameters $N_{\max }$. This shows good convergence up to a scale proportional to $\sqrt{N_{\max }}$. This figure clearly shows that the TDA in this model falls off faster than $1 / k_{\perp}^{2}$. Our best fit for the asymptotic behavior is

$$
k_{\perp} \phi_{\perp}\left(\vec{k}_{\perp}\right) \sim \mathrm{e}^{-c k_{\perp}},
$$

which suggests that the integral for the decay constant is finite, even in the limit of a complete basis. As indicated before, we may need alternative computational tools to accurately determine the asymptotic behavior of the TDA, and whether this behavior follows from the one-gluon exchange, or from the one-gluon exchange in combination with our specific model for the transverse and longitudinal confinement.
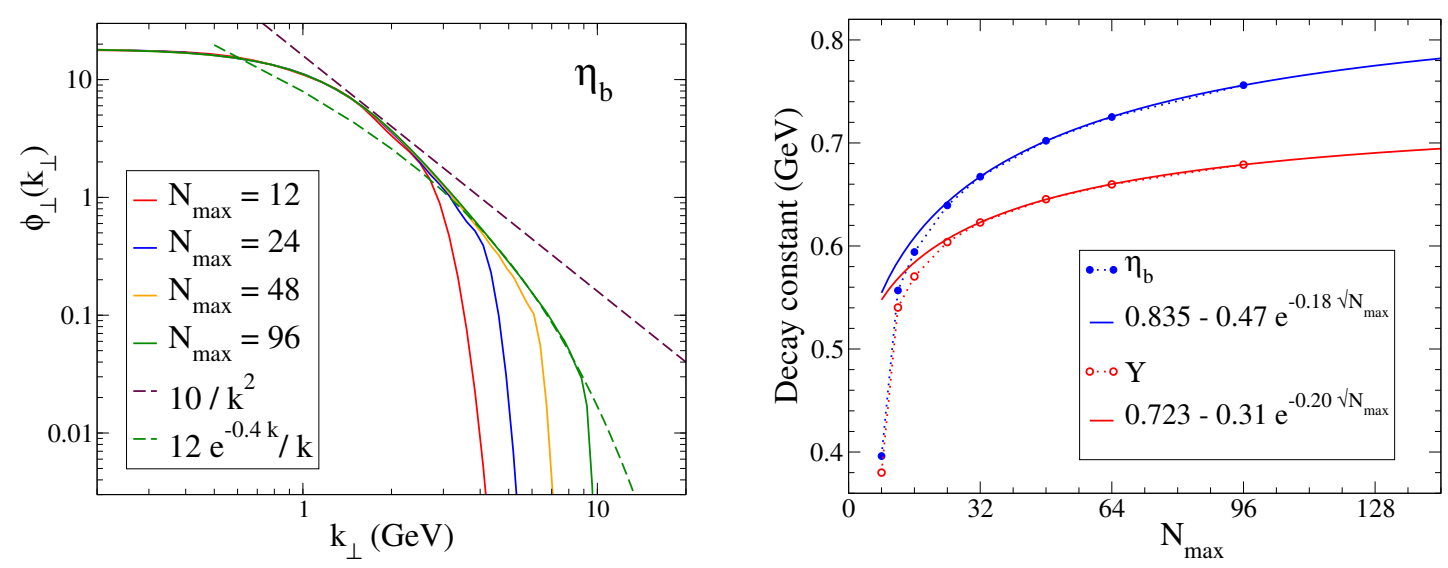

Figure 3: Transverse DA for $\eta_{b}$ (left) and extrapolation of the decay constants (right). 
Inspired by this behavior, we extrapolate the decay constants by a fit of our finite basis results

$$
f\left(N_{\max }\right)=f\left(N_{\max }=\infty\right)+a \mathrm{e}^{-c \sqrt{N_{\max }}},
$$

as is shown in the right panel of Fig. 3 for the $\eta_{b}$ and $\Upsilon$. This extrapolation also works quite well for the decay constants of the $\eta_{c}, J / \Psi$, and $B_{c}$. Of course, these extrapolated decay constants are significantly larger than those reported in Refs. [2,9], which were truncated at a finite scale.

\section{LFWF of unequal-mass heavy mesons}

For equal-mass constituents, the LFWF $\psi\left(\vec{k}_{\perp}, x\right)$ has its maximum value at $x=\frac{1}{2}$ for all $k_{\perp}$, but for systems with valence quarks of unequal masses such as $B_{c}$, this is not the case. Nonperturbatively, one expects this maximum to occur at $\frac{m_{Q}}{m_{Q}+m_{q}}$; however, the left panel of Fig. 4 clearly shows that this maximum depends on $k_{\perp}$. At $k_{\perp}=0$, the peak-position (indicated by an open circle) is at $x>\frac{m_{b}}{m_{b}+m_{c}}$, but for increasing $k_{\perp}$, this maximum occurs at lower values of $x$. In the limit $k_{\perp} \rightarrow \infty$ the effect of the unequal masses becomes negligible, and the maximum position approaches $x=\frac{1}{2}$.

With the LFWF interpreted as the probability amplitude, its peak coincides with the maximum of the probability distribution for finding the system with in a given momentum configuration. This momentum-space probability density can be alternatively calculated using the light-front parton gas model [10]. Specifically, the joint probability distribution in $k_{\perp}$ and $x$ is given by

$$
\rho\left(k_{\perp}, x\right) \sim \delta\left(\frac{k_{\perp}^{2}+m_{Q}^{2}}{x}+\frac{k_{\perp}^{2}+m_{q}^{2}}{1-x}-u\right),
$$

where the quantity $u$ is the available thermal energy for the relative motion of the valence quarks, after averaging interactions other than the light-front kinetic energy. The parameters $m_{Q}$ and $m_{q}$ need not to be identical to those in the light-front Hamiltonian because of this averaging - one can think of these parameters as 'effective masses'.

Notice that with fixed $x$, the peak of the distribution always locates at $k_{\perp}=0$. The peak of the LFWF with fixed $k_{\perp}$ is given by the $x$ which makes the partial derivative of Eq. (3.1) with respect
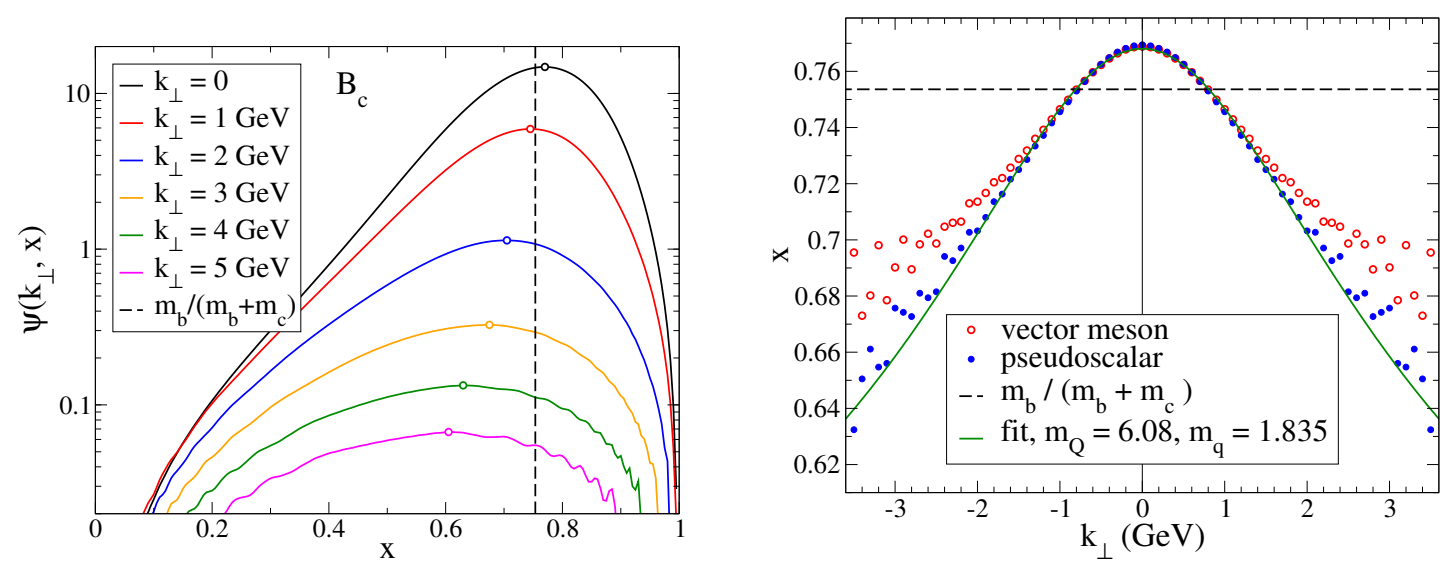

Figure 4: Dominant component $\psi_{\uparrow \downarrow-\downarrow \uparrow}\left(x, k_{\perp}\right)$ of the LFWF for $B_{c}$ at fixed $k_{\perp}$ (left) and position of the maximum in $x$ in the LFWF at fixed $k_{\perp}$ as a function of $k_{\perp}$ (right), with the fit Eq. (3.2). 
to $x$ vanish. The derivative of the $\delta$-function can be understood by approximating it with a narrow gaussian function. Explicitly, we obtain for the peak-location in $x$ at fixed $k_{\perp}$

$$
x=\left(1+\sqrt{\frac{k_{\perp}^{2}+m_{q}^{2}}{k_{\perp}^{2}+m_{Q}^{2}}}\right)^{-1},
$$

which indeed describes the peak-position in our model, see the right panel of Fig. 4, with the same effective masses $m_{Q}$ and $m_{q}$ for the lowest pseusoscalar and vector states. As a consequence, different light-front observables such as the Distribution Amplitude (DA) and the Parton Distribution Function (PDF) have their maximum at different values of $x$. This effect is more pronounced as the mass difference between the quarks becomes larger [11].

\section{Acknowledgements}

This work was supported by the US Department of Energy under Grants No. DE-FG0287ER40371 and No. DE-SC0018223 (SciDAC-4/NUCLEI). Compuutational resources were provided by the National Energy Research Scientific Computing Center (NERSC), which is a US Department of Energy Office of Science user facility, supported under Contract No. DE-AC02$05 \mathrm{CH} 11231$.

\section{References}

[1] Y. Li, P. Maris, X. Zhao and J.P. Vary, Heavy Quarkonium in a Holographic Basis, Phys. Lett. B 758, 118 (2016) [arXiv:1509.07212 [hep-ph]].

[2] Y. Li, P. Maris and J.P. Vary, Quarkonium as a relativistic bound state on the light front, Phys. Rev. D 96, 016022 (2017) [arXiv:1704.06968 [hep-ph]].

[3] S.J. Brodsky, G.F. de Teramond, H.G. Dosch and J. Erlich, Light-Front Holographic QCD and Emerging Confinement, Phys. Rept. 584, 1 (2015) [arXiv:1407.8131 [hep-ph]].

[4] M. Li, Y. Li, P. Maris and J.P. Vary, Radiative transitions between $0^{-+}$and $1^{--}$heavy quarkonia on the light front, Phys. Rev. D 98, 034024 (2018) [arXiv:1803.11519 [hep-ph]].

[5] M. Li, Frame dependence of transition form factors in light-front dynamics, these proceedings, arXiv:2001.02108 [nucl-th].

[6] G. Chen, Y. Li, K. Tuchin and J.P. Vary, Heavy quarkonia production at energies available at the CERN Large Hadron Collider and future electron-ion colliding facilities using basis light-front quantization wave functions, Phys. Rev. C 100, 025208 (2019) [arXiv:1811.01782 [nucl-th]].

[7] M. Tanabashi et al., Review of Particle Physics, Phys. Rev. D 98, 030001 (2018).

[8] P. Maris, C.D. Roberts and P.C. Tandy, Pion mass and decay constant, Phys. Lett. B 420, 267 (1998) [arXiv:nucl-th/9707003].

[9] S. Tang, Y. Li, P. Maris and J.P. Vary, $B_{c}$ mesons and their properties on the light front, Phys. Rev. D 98, 114038 (2018) [arXiv:1810.05971 [nucl-th]].

[10] S. Jia and J.P. Vary, Parton distribution functions from scalar light front parton gas model, arXiv:1812.09340 [nucl-th].

[11] S. Tang, Y. Li, P. Maris and J.P. Vary, Heavy-Light Mesons on the Light Front, arXiv:1912.02088 [nucl-th]. 